\title{
A Theoretical Analysis of How Segmentation of Dynamic Visualizations Optimizes Students' Learning
}

\author{
Ingrid A. E. Spanjers • Tamara van Gog • \\ Jeroen J. G. van Merriënboer
}

Published online: 13 May 2010

(C) The Author(s) 2010. This article is published with open access at Springerlink.com

\begin{abstract}
This article reviews studies investigating segmentation of dynamic visualizations (i.e., showing dynamic visualizations in pieces with pauses in between) and discusses two not mutually exclusive processes that might underlie the effectiveness of segmentation. First, cognitive activities needed for dealing with the transience of dynamic visualizations impose extraneous cognitive load, which may hinder learning. Segmentation may reduce the negative effect of this load by dividing animations into smaller units of information and providing pauses between segments that give students time for the necessary cognitive activities after each of those units of information. Second, event segmentation theory states that people mentally segment dynamic visualizations during perception (i.e., divide the information shown in pieces). Segmentation of dynamic visualisation could cue relevant segments to students, which may aid them in perceiving the structure underlying the process or procedure shown.
\end{abstract}

Keywords Dynamic visualizations · Animations · Segmentation · Cognitive load $\cdot$ Learning

Dynamic visualizations such as videos and animations are increasingly used in instructional materials to visualize complex natural processes (e.g., the formation of lightning; Mayer \& Chandler 2001), mechanical systems (e.g., an electric motor; Mayer et al. 2003), and different kinds of procedures (e.g., solving probability calculation problems, Spanjers et al., 2010; or first aid: Arguel \& Jamet 2009). Dynamic visualizations are seen as attractive for students (e.g., Chandler 2009) and are usually expected to be more effective than static

I. A. E. Spanjers · J. J. G. van Merriënboer

Department of Educational Development and Research, School of Health Professions Education, Maastricht University, P.O. Box 616, 6200 MD Maastricht, The Netherlands

I. A. E. Spanjers $(\bowtie) \cdot$ T. van Gog $\cdot$ J. J. G. van Merriënboer

Centre for Learning Sciences and Technologies, Netherlands Laboratory for Lifelong Learning,

Open Universiteit in The Netherlands, Heerlen, The Netherlands

e-mail: i.spanjers@EDUC.unimaas.nl

T. van Gog

Institute of Psychology, Erasmus University Rotterdam, Rotterdam, The Netherlands 
pictures because students can perceive changes over time rather than having to mentally infer them (e.g., Hegarty et al. 2003; Lowe 1999). The general idea is that compared to having to infer or imagine changes, perceiving changes frees up cognitive resources (Hegarty et al.2003; Schnotz \& Rasch 2005). However, research has shown that complex dynamic visualizations are not always more effective for learning than static pictures (e.g., Hegarty et al. 2003; Mayer et al. 2005; Tversky et al. 2002), although for certain types of tasks, dynamic visualizations seem to be more effective, especially demonstrations of procedures involving human movement (Höffler \& Leutner 2007; see also Van Gog et al. 2009).

To improve the effectiveness of complex dynamic visualizations, several design measures have been proposed (for reviews, see Wouters et al. 2008; Wouters et al. 2007). Some of these measures involve the manipulation of characteristics of dynamic visualizations. De Koning et al. (2009) reviewed ways to manipulate visuospatial characteristics, such as visually highlighting certain parts of the dynamic visualization to fix students' attention on those parts. Next to visuospatial characteristics, temporal characteristics can be manipulated. For example, by segmentation, that is, showing dynamic visualizations in segments. A segment is a piece of the dynamic visualization that has an identifiable begin and end point.

Several studies examined the effects of segmentation of complex dynamic visualizations on learning. In these studies, the segments were distinguished by inserting pauses between them (Boucheix \& Guignard 2005; Hasler et al. 2007; Mayer \& Chandler 2001; Mayer et al. 2003; Moreno 2007; Spanjers et al., 2010). Boucheix and Guignard (2005) compared the effects on learning from different versions of a slideshow about gearing systems, consisting of two wheels and a motor: a version in which students were able to start the next slide or repeat the previous slide and a fast and slow version without learner control. They found larger gains from pretest to posttest for students who could start the next slide or repeat the previous one. In separate analyses for groups differing in prior knowledge, the effect was only significant in the low prior knowledge group (i.e., an expertise reversal effect; Kalyuga 2007). In the multimedia learning environment of Mayer et al. (2003), an animated pedagogical agent explained the functioning of an electric motor with some animations. In one condition, students could decide at the end of each segment when to continue with the next segment as well as which segment to continue with or to repeat the animation (if there was one). In the other condition, the same material was shown in a continuous presentation. They found that students who studied the segmented learning environment performed better on a transfer test, in which they had to use what they learned to answer novel questions, than students in the other condition. Although these studies provide some evidence that segmentation might be beneficial, the effects may have been influenced by the learner control options that students were given in these studies (e.g., the possibility to repeat slides or animations and/or to select the next segment) and the design of the learning materials (e.g., a division in different slides).

In the studies of Moreno (2007), Mayer and Chandler (2001), and Hasler et al. (2007), some learner control was also present in combination with segmentation, although to a lesser extent: the dynamic visualisations stopped automatically at the end of each segment, and the participants could decide when they wanted to continue with the next segment. Moreno (2007) found that participants who studied a segmented version of an exemplary classroom video (experiment 1) or animation (experiment 2) in which teaching skills were modeled remembered more of the modelled behaviour, reported to invest less mental effort, and experienced the learning materials as less difficult than participants who studied nonsegmented versions of the material. Mayer and Chandler (2001) found that students who 
learned with a segmented animation on the formation of lightning performed better on a transfer test than students who learned with a non-segmented animation. Hasler et al. (2007) compared four versions of their learning material on the causes of day and night: a segmented animation, a non-segmented animation that students could pause at each moment (i.e., with learner control), a non-segmented animation without learner control, and a non-segmented audio-only version without learner control. Learning time was equalized for the conditions by having students study the learning material repeatedly until $10 \mathrm{~min}$ were over. Their results showed that learners who studied the segmented animation or the animation that they could pause performed better on difficult test questions than students who studied one of the two other versions of the material, even though most learners who could pause the animation did not use that option. Although learners in these studies by Moreno (2007), Mayer and Chandler (2001), and Hasler et al. (2007) had less control than the learners in the studies of Boucheix and Guignard (2005) and Mayer et al. (2003), learner control might still have influenced the effects of segmentation that were found.

In the study by Spanjers et al. (2010), segmentation was not combined with learner control. They investigated the effects of segmented and non-segmented animations on probability calculation procedures, and their segmented animations automatically paused after each segment and automatically continued after 2 s. A significant interaction was found between the effects of segmentation and prior knowledge: Students with lower levels of prior knowledge learned more efficiently from segmented animations than from nonsegmented animations, while students with higher levels of prior knowledge learned equally efficiently from non-segmented and segmented ones (i.e., an expertise reversal effect; Kalyuga 2007).

These studies show that, at least for novices, segmentation may have positive effects on learning from dynamic visualizations. But how can this effect be explained, or in other words, what cognitive processes underlie this effect? We propose that two possible though not mutually exclusive processes might be responsible: (1) segmentation reduces the high cognitive load associated with information transience due to the fact that the dynamic visualization is divided into smaller units of information and is paused several times that yields extra time for processing those units, and (2) segmentation breaks the dynamic visualization down into meaningful pieces, which may foster students' understanding of the underlying structure of the depicted process or procedure. These processes will be discussed in more detail in the next sections.

\section{Reducing Information Transience}

One main cause for why learning from dynamic visualizations is often not more effective than learning from static pictures is that information in dynamic visualizations is usually transient; that is, information is continuously replaced with new information, and what is visible now makes way for other information presented in the next moment (Ayres \& Paas 2007). Learning involves the construction of cognitive schemas, which are stored in longterm memory. To construct those schemas, information from the dynamic visualizations needs to be maintained and processed in working memory (Sweller et al. 1998). That is, information elements need to be selected from the stream of information and then need to be mentally integrated with information that was presented earlier and with prior knowledge in order to form a representation from the shown information (Moreno \& Mayer 2007). Therefore, the limitations of working memory pose a bottleneck for learning (Sweller 2010; Sweller et al. 1998). 
The time-based resource sharing model of working memory (Barrouillet \& Camos 2007) states that the focus of attention in working memory is capacity limited, which implies that attention can be directed at only one cognitive activity at a time. Maintaining information (i.e., refreshing memory traces) and processing information both require attention, which has to be shared between these cognitive activities by rapidly and frequently switching attention from one activity to the other. Cognitive load therefore depends not only on the number of processes that are competing for attention but also on how fast processing needs to be done (e.g., in a continuous dynamic visualization, information keeps coming in at a very high pace) and how long the processing takes. Under conditions of high cognitive load, some cognitive activities require attention during such a large proportion of the available time that insufficient time remains for attending to other cognitive activities.

Cognitive load theory (Sweller 2010; Sweller et al. 1998; Van Merriënboer \& Sweller 2005) makes a distinction between cognitive activities imposing intrinsic cognitive load and cognitive activities imposing extraneous cognitive load. Maintaining and processing information elements inherent to the learning content and the relations between those elements impose intrinsic cognitive load on working memory. The higher the number of information elements and relations between them, the higher the intrinsic cognitive load and the more difficult the material is to learn. For example, an animation in which the names of the different parts of an eye are sequentially presented is less complex than an animation in which the changes occurring during accommodation of the eyes to see approaching objects clearly are presented because in the former animation, the different parts can be learned sequentially and in a relatively isolated manner, while in the latter, the relations and interactions between different parts of the eye need to be learned as well. But the number of interrelated information elements is not the sole determinant of intrinsic cognitive load. Students' prior knowledge influences what constitutes a single information element. As mentioned before, learning involves the construction of cognitive schemas, and a schema can be handled in working memory as one single information element. Therefore, the same learning materials impose less intrinsic load for students with higher levels of prior knowledge than for students with lower levels of prior knowledge or no prior knowledge (Sweller et al. 1998). Under conditions of very high intrinsic cognitive load, the number of information elements and their relations that have to be maintained and processed in working memory can be so high that insufficient time is available for attending to maintenance and processing of all the elements and relations. Therefore, the cognitive load theory recommends that intrinsic load of learning materials is optimized, that is, at a difficulty level that is challenging but not too high (Sweller 2010; Schnotz \& Kürschner 2007). Optimizing cognitive load implies that one decreases the complexity of the material if the amount of intrinsic load might overload the learner's limited working memory, for example, by requiring learners first to learn the information elements inherent to the learning material without learning their relations and subsequently requiring them to learn the relations between the previously learned information elements. It also implies increasing the complexity of the material, if learners do not fully use their working memory resources, for example, by requiring that learners not only learn a particular procedure but also under which conditions they have to use that particular procedure (Sweller 2010). In other words, one has to use tasks that are in the learner's zone of proximal development (Vygotsky 1978; see also Schnotz \& Kürschner 2007).

Extraneous cognitive load is imposed by maintaining and processing activities that result from a non-optimal design of learning materials (Sweller 2010; Sweller et al. 1998). For example, to learn from dynamic visualizations, information from different points in time needs to be mentally integrated, and when information is transient, this requires that 
information from a certain point in time is maintained in working memory and then linked to information presented later (Lowe 1999). Moreover, new incoming information needs to be processed while maintaining the previously presented information (e.g., Ayres \& Paas 2007; Moreno \& Mayer 2007). Such cognitive activities that impose extraneous load reduce the time available for maintaining and processing activities that impose intrinsic cognitive load, and as a consequence, learning may be hampered because insufficient time is left for attending to these intrinsic maintenance and processing activities. This will happen especially when animations are high in intrinsic load; when they are low in intrinsic load, there may be sufficient time to attend to both processes that impose intrinsic and processes that impose extraneous load. Therefore, on complex dynamic visualizations that are high in intrinsic load, the need to attend to processes imposing extraneous load needs to be decreased as much as possible so that learning will not be hampered (Sweller 2010; Sweller et al. 1998).

In segmented dynamic visualizations, the stream of information is divided into smaller units, and pauses between segments provide students with sufficient time to attend to necessary cognitive activities on the bit of information presented in the previous segment, without having to simultaneously attend to new incoming information (e.g., Mayer \& Moreno 2003; Moreno \& Mayer 2007). Thus, segmentation reduces the harmful effects of information transience in dynamic visualizations by reducing the co-occurrence of processing activities, thereby reducing cognitive load at certain points in time (e.g., Ayres \& Paas 2007; Mayer \& Moreno 2003; Moreno \& Mayer 2007; Schnotz \& Lowe 2008). It should be noted that by providing pauses, segmentation does increase the total available learning time. Yet, this does not imply that increased learning time causes the positive effects on learning. It can be questioned, for example, whether providing additional time at the end of an entire, continuous dynamic visualization would enhance learning because this would do nothing to alleviate the high load at certain points in time. Support for the claim that the segmentation effect is not simply due to differences in learning time is provided by the study of Hasler et al. (2007), in which learning time was equal for all conditions but segmentation still affected learning outcomes.

Next to the assumption that giving students extra time for necessary cognitive processes may have positive effects on learning, segmentation may also support learning by highlighting the structure underlying the process or procedure shown in dynamic visualizations.

\section{Enhancing Perception of the Underlying Structure}

In many dynamic visualizations multiple subevents or substeps in a process or procedure are shown across time (Meyer et al. 2010; Schnotz \& Lowe 2008). For example, in the case of a four-stroke engine, one can divide its functioning in several substeps such as intake stroke, compression stroke, etc. (Meyer et al. 2010). People also tend to perceive events or procedures shown in dynamic visualizations as consisting of a series of discrete subevents or substeps (e.g., Kurby \& Zacks 2008; Zacks et al. 2007). This implies that they mentally segment the continuous stream of information presented. The event segmentation theory (Zacks et al. 2007) describes the processes underlying mental segmentation. It is assumed that people form event models in working memory on the basis of incoming sensory information and prior knowledge. Based on these models, they form predictions about what will happen in the next moment and compare these predictions with what actually happens according to the new incoming sensory information. When the predictions and the new 
incoming sensory information differ too much, a new event model for the next segment needs to be constructed. At this point, a so-called event boundary is distinguished (Zacks et al. 2007). Physical changes in the information shown, such as changes in movements, speed, or distance between objects as well as structural changes, such as subgoal completion and initiation, lead to a decrease in predictability of the new incoming sensory information and therefore to the distinction of event boundaries (Kurby \& Zacks 2008; Zacks 2004; Zacks et al. 2007).

For example, if a general physician in a video about putting on walking plaster starts winding a layer of cotton around the patient's leg, one can predict that he or she will continue with that action until the patient's leg is wrapped up in a layer of cotton (Robroek $\&$ Van de Beek 2005). If the patient's leg is wrapped up in a layer of cotton, the coherent movement pattern of the general physician changes and another subgoal will be initiated, with the consequence that it is more difficult to predict the new incoming sensory information and an event boundary is distinguished (Zacks et al. 2007). Because the occurrence of discontinuities in the incoming sensory information and the completion and the initiation of subgoals are related to each other (although for some subevents, more tightly than for others), mental segmentation on the basis of discontinuities in the incoming sensory information supports interpretation and therefore comprehension of the information shown in terms of subgoals (Baldwin et al. 2001; Hard et al. 2006; Zacks 2004). At event boundaries, the representations in working memory are updated. Because more extensive processing of information takes place at event boundaries than between event boundaries, more information about those boundaries is stored in long-term memory than about the parts between the boundaries (Zacks et al. 2007). Finally, segmentation aids in chunking information, which reduces the cognitive load imposed by maintaining the information (Kurby \& Zacks 2008; Schnotz \& Lowe 2008).

Since mental segmentation is in this way related to interpretation and storage in longterm memory, it can be expected that individual differences in mental segmentation lead to differences in learning outcomes (cf. Koopman \& Newtson 1981). Differences in segmentation rates have been found between participants who were asked to segment videos about several human activities (e.g., a couple playing a party game, a person doing dishes, assembling a saxophone, and fertilizing house plants); that is, there were differences in the size of the substeps or subevents in which participants divided the videos (Hanson \& Hirst 1989; Zacks et al., 2006; Zacks et al., 2001). Those differences have been shown to influence the recall of details of videos and memory for the temporal order of subevents or substeps shown. Participants who divided videos about a couple playing a game, a person cleaning a pistol, or a person upgrading a computer in smaller subevents recalled more subevents on a free recall test (Hanson \& Hirst 1989; Schwan et al. 2000). Zacks et al. (2006) did not examine free recall but assessed performance on a test requiring participants to sort pictures taken from videos of planting flowers in a window box, setting up a tent, washing clothes, and washing a car in the correct order. Their second experiment showed that requiring participants to segment in smaller subevents was associated with worse performance on the test than requiring them to segment in larger subevents. They suggest that segmentation in smaller subevents might have led to a focus on temporal relations between small subevents, which may have negative effects on memory of the more global temporal relations. Zalla et al. (2003) found that individuals with frontal lobe damage had difficulties with segmenting videos, depicting a person performing actions in an office or a couple playing a party game, in larger subevents. Although they recalled the same number of events from the videos as controls without brain lesions on a free recall test, they tended to recall the subevents without links between them and in a wrong temporal order. Like the 
second experiment by Zacks et al. (2006), this study suggests that segmentation in smaller subevents may be associated with difficulties in remembering the temporal order of subevents. Although the dynamic visualizations used in these studies depicted everyday life activities, they might have some relevance for education; consider for example dynamic visualizations on learning to perform first aid procedures (Arguel \& Jamet 2009). In addition, Zacks et al. (2006; experiment 2) had some participants perform the test without having seen the videos, and their scores suggest that the temporal order was not completely arbitrary or commonsense. The order of segments of a process or procedure is relevant in many instructional dynamic visualizations as well. For example, in the study by Moreno and Valdez (2005), the learning task consisted of putting segments of an animation about lightning formation in the correct temporal and causal order.

Concerning instructional dynamic visualizations, these findings might imply that differences in learning outcomes may occur between students, depending on how they mentally segmented the dynamic visualization (cf. Koopman \& Newtson 1981). Further evidence for an effect of mental segmentation differences on learning is provided by studies about design measures that likely disturbed participants' perception of the structure underlying the information shown (Kurby \& Zacks 2008; Zacks et al. 2007). Schwan and Garsoffky (2004) showed participant videos about cleaning a pistol or upgrading a computer, either unaltered, with event boundaries replaced by a 1-s black screen or with parts between event boundaries replaced by a 1-s black screen. The event boundaries had been determined by studying which locations experts indicated as such (see also Schwan et al. 2000). It was hypothesized that deleting event boundaries disrupts the event structure people use for their mental segmentation. Indeed, participants viewing a video from which event boundaries were deleted indicated the ends of segments about equally often at event boundaries as at points between event boundaries, whereas participants viewing other versions of the video indicated ends of segments about twice as often at event boundaries than at points between event boundaries. Moreover, participants who viewed the video from which event boundaries had been deleted recalled a lower proportion of the subevents than the participants from the other groups, which might be due to disruption of the event structure, reduced coherence of the video, and a lower proportion of relevant steps shown. Between the unaltered version of the video and the version with replacements between event boundaries, only small differences were found in the proportion of recalled subevents, but different subevents were recalled. Schwan and Garsoffky (2004) proposed that these differences are due to changes in the temporal density of subevents that are the consequence of the deletion of parts. Since deletions and insertions of black screens always co-occurred, it is not clear whether the insertion of black screens alone would affect learning.

Boltz (1992) showed participants an episode of a mini-series without commercials or with commercials inserted either at event boundaries or between event boundaries. The locations of event boundaries had also been determined based on a previous study. She hypothesized that insertion of commercials between event boundaries would hinder perceiving the underlying event structure of the episode. Her results were in line with this hypothesis: Participants who saw this version recalled less from the episode than participants who saw another version. In addition, a positive effect on recall was found of inserting twice as many commercials at event boundaries and a negative effect of inserting twice as many commercials between event boundaries.

If design measures can hinder participants to perceive the underlying structure of a depicted event, process, or procedure, it is likely that they can be applied to aid perceiving that structure as well. When design measures make event boundaries more salient, they have a temporal cueing effect (Boltz 1992; Schwan et al. 2000; Zacks et al. 2007). 
Temporal cueing involves highlighting points in time rather than visuospatial parts. A number of studies provide evidence that temporal cueing of event boundaries enhances remembering of information shown. Schwan et al. (2000) highlighted either event boundaries or points between event boundaries through cuts in videos about cleaning a pistol or upgrading a computer. It was found that cueing event boundaries prevented the occurrence of a negative relation between segmentation in smaller subevents and completeness (although not detailedness) of recall. Additionally, it was found that time periods around event boundaries that were cued by a cut were recalled better than around event boundaries that were not cued. Arguel and Jamet (2009, experiment 1) compared three versions of learning materials on first aid: dynamic visualizations combined with key frames (i.e., static pictures from different substeps), dynamic visualizations without key frames, and key frames without dynamic visualizations. The addition of the key frames to the dynamic visualization may have served as a cue of the underlying structure of the shown procedures, and this condition had better posttest performance than the other conditions. In experiment 1 , all key frames were revealed at once, right at the beginning of the dynamic visualizations. In their second experiment, Arguel and Jamet (2009) showed that revealing the key frames sequentially (after which they remained visible) led to better posttest performance than presenting them at once at the beginning of the dynamic visualization. When twice as many frames as in experiment 1 were presented, this had a negative effect on posttest performance. This negative effect tended to disappear if the frames were presented sequentially. In addition to cueing the underlying event structure, presentation of key frames may aid in maintaining and chunking the shown information as well. Well-chosen key frames provide external representations of previous substeps, so that students do not have to use a proportion of the available time to refresh memory traces to be able to mentally integrate them with information presented later (Bétrancourt et al. 2008). They also provide students with a compact presentation of the substeps so that students are aided in chunking the information (Schnotz \& Lowe 2008). In other words, they lead to a reduction in cognitive activities imposing extraneous cognitive load (Arguel \& Jamet 2009; Schnotz \& Lowe 2008) and an increase in cognitive activities associated with learning.

Segmentation of instructional dynamic visualizations is another design measure that may cue event boundaries. That is, rather than relying on students' ability to mentally segment the dynamic visualisations, this is done for them. Segmentation might enhance learning by aiding students in perceiving the underlying structure of the process or procedure and by aiding chunking. This function of segmentation is suggested by the work of Catrambone on learning to solve probability calculation problems from worked examples $(1995,1996$, and 1998). For example, Catrambone (1995) compared four groups, which differed on whether or not a label for a particular calculation substep was provided (i.e., providing meaning to the step) and on whether or not that calculation substep was placed on a separate line (i.e., cue of what constituted a step). Learning outcomes were higher, and students mentioned a substep more often in their description of the calculation procedure if a label was provided, the step was visually isolated or both the label was provided and the step was isolated, compared with a control condition in which no cues were provided. Segmentation of dynamic visualizations might have a similar effect. That is, by temporally cueing event boundaries, segmentation indicates which substeps or subevents belong together and which steps need to be separated. This may also encourage students to self-explain why particular substeps or subevents belong together or need to be separated, which might lead to deeper understanding of the process or procedure (Catrambone 1995, 1996, 1998; see also Wouters et al. 2008). 
Students with higher levels of prior knowledge might not need these temporal cues or might even be hampered by them. The findings of Boucheix and Guignard (2005) and Spanjers et al. (2010) show that students with higher levels of prior knowledge do not need additional guidance through segmentation. For students with higher levels of prior knowledge, the amount of cognitive resources they can devote to cognitive activities with a positive effect on learning is reduced when they have to reconcile the instructional guidance with the guidance given by their available cognitive schemas (Kalyuga 2007).

\section{Discussion}

This article proposed that two processes may underlie the effectiveness of segmentation of dynamic visualizations. First of all, in the studies on segmentation that were conducted thus far, segmentation was always associated with pauses between segments. These pauses may have caused the beneficial effects on cognitive load and learning because they give students time to perform the cognitive activities needed for learning on a smaller unit of information, without having to simultaneously attend to the next unit of information (e.g., Mayer \& Moreno 2003: Moreno \& Mayer 2007). This first explanation is mainly based on cognitive load theory (Sweller et al. 1998), although we also introduced a perspective on cognitive load based on the work by Barrouillet and Camos (2007) that is new to cognitive load theory. Second, segmentation may be effective because it cues students to perceive event boundaries, thereby aiding them in perceiving the structure underlying the process or procedure shown (cf. Catrambone 1995, 1996, 1998; see also Wouters et al. 2008). This second explanation is mainly based on event segmentation theory (Zacks et al. 2007). The two explanations are thus based on two different theories about processes involved in learning from dynamic visualisations. As mentioned before, the explanations are not mutually exclusive, and there are also some connections between the theories. For example, mental segmentation, which is the process on which the event segmentation theory focuses, influences the representations held in working memory (e.g., Zacks et al. 2007) that influence cognitive load.

Which of the two mechanisms, that is, pauses or temporal cueing, plays the most important role in explaining the positive effects of segmentation on learning or whether their combination is crucial cannot be judged based on previous studies in which a pause also automatically cued the end of a segment. Therefore, this remains an important empirical question for future studies in which different versions of instructional dynamic visualizations with segmentation through pauses, cues, or both could be compared.

Next to this central question, there are some other interesting directions for future research on the effects of segmentation of dynamic visualizations on cognitive load and learning. The first is whether effects of segmentation (or more specifically, of pauses and temporal cueing) would differ between different types of dynamic visualizations. Studies based on the event segmentation theory often used dynamic visualizations depicting everyday life activities, and naturally, all participants have experience with observing such activities (Hard et al. 2006; Zacks et al. 2007). Moreover, in some studies, some or even all of the depicted events were familiar to the participants (Hanson \& Hirst 1989; Zacks et al. 2006; Zacks et al. 2001). Consequently, participants would already have relevant cognitive schemas for those events that would influence the construction of event models (Zacks et al. 2007); one might hypothesize that, perhaps, less cognitive load is required for the construction of event models in such visualizations of everyday tasks than in visualizations on, for example, natural or biological processes or on the spatial arrangement of complex 
chemical molecules and other structures. Furthermore, depictions of familiar human movements seem to automatically trigger activation of the mirror neuron system with the consequence that an effortless process of embodied simulation occurs (Van Gog et al. 2009), and mirror neurons have been suggested to be involved in the process of mental segmentation as well (Sommerville \& Decety 2006). Therefore, differences in ease of mental segmentation and in the amount of cognitive load imposed by mental segmentation may exist between dynamic visualizations depicting human movement activities and those depicting other kinds of processes, procedures, or structures, and although segmenting dynamic visualizations might be effective for both types of visualizations, it might be most crucial for non-human movement activities if these indeed impose higher load. Whether the cognitive load imposed by constructing event models is lower in human movement tasks than in non-human movement tasks could be investigated with online physiological measures of cognitive load, such as pupil dilation (Kahneman \& Beatty 1966; Van Gerven et al. 2004) or EEG (Antonenko et al. 2010). For example, Swallow and Zacks (2004) found transient small increases in participants' pupil dilation after event boundaries in dynamic visualisations.

A second direction for future research concerns the role of learner characteristics, such as prior knowledge that may interact with the effects of segmentation. In this article, we described two studies (Boucheix \& Guignard 2005; Spanjers et al., 2010) that found an expertise reversal effect of segmentation on cognitive load and/or learning. However, before concluding that segmentation per se is not helpful for students with higher levels of prior knowledge, we might need to investigate the effects of different grain sizes of segments. There are no clear guidelines on how to determine the length of a segment; some researchers base the length on theories with regard to cognitive functioning (Hasler et al. 2007; Mayer \& Chandler 2001; Moreno 2007), while others consult experts in order to determine what meaningful pieces are in which to divide the dynamic visualizations (Arguel \& Jamet 2009; Schwan \& Garsoffky 2004; Schwan et al. 2000; Spanjers et al., 2010). The study by Boltz (1992) suggested that segment length may influence learning: She found a positive effect on recall of dividing dynamic visualizations in shorter segments by inserting twice as many commercials at event boundaries. The question is, however, whether the effects on cognitive load and learning of different segment lengths would be the same for low and high prior knowledge learners. It might be that novices need smaller segments because all information is new to them, whereas learners with more prior knowledge might benefit from larger segments. In addition, it might be interesting to investigate whether students with different levels of prior knowledge would benefit from actively segmenting dynamic visualizations themselves. Interactivity with dynamic visualizations has been proposed as a way to encourage students to engage in activities contributing to learning (Wouters et al. 2007). If students must segment dynamic visualisations themselves, they are encouraged to focus on which subevents or substeps are depicted, which may have a positive effect on learning. However, this positive effect is probably found only when cognitive resources are available for attending to this additional task while learning the content of the visualization (i.e., if students devote all their attention to determining segments, they might learn very little). Therefore, actively segmenting dynamic visualisations may only be beneficial for students with higher levels of prior knowledge. This kind of research on the effects of having learners segment animations themselves could perhaps also shed light on the required grain size of segments for different types of learners by providing indications on whether students with lower and higher prior knowledge would distinguish smaller or larger segments. The empirical findings from previous studies on mental segmentation with regard to this 
question are mixed. Participants in the study of Hard et al. (2006) who had seen the dynamic visualizations a number of times before they had to segment them, divided them in larger units than participants who had not seen the dynamic visualizations before. However, other studies found no effect of expertise, training, or familiarity with the content on the length of segments made by participants (Schwan et al. 1998; Zacks et al. 2001). Finally, next to prior knowledge, there may be other learner characteristics that influence the effects of segmentation on learning, such as motivation, spatial ability, or age.

In conclusion, two processes may underlie the effectiveness of segmented dynamic visualizations: pausing and temporal cueing. Future empirical studies should further examine these cognitive processes and associated cognitive load as well as factors influencing these processes.

Acknowledgments This work was funded by The Netherlands Organization for Scientific Research (NWO; project No. 400-07-017).

Open Access This article is distributed under the terms of the Creative Commons Attribution Noncommercial License which permits any noncommercial use, distribution, and reproduction in any medium, provided the original author(s) and source are credited.

\section{References}

Antonenko, P., Paas, F., Grabner, R., \& Van Gog, T. (2010). Using electroencephalography to measure cognitive load. Educational Psychology Review (this issue).

Arguel, A., \& Jamet, E. (2009). Using video and static pictures to improve learning of procedural contents. Computers in Human Behavior, 25, 354-359.

Ayres, P., \& Paas, F. (2007). Making instructional animations more effective: A cognitive load approach. Applied Cognitive Psychology, 21, 695-700.

Baldwin, D. A., Baird, J. A., Saylor, M. M., \& Clark, M. A. (2001). Infants parse dynamic action. Child Development, 72, 708-717.

Barrouillet, P., \& Camos, V. (2007). The time-based resource-sharing model of working memory. In N. Osaka, R. H. Logie, \& D. E. Mark (Eds.), The cognitive neuroscience of working memory (pp. 57-80). Oxford: Oxford University Press.

Bétrancourt, M., Dillenbourg, P., \& Clavien, L. (2008). Display of key pictures from animation: Effects on learning. In J.-F. Rouet, R. Lowe, \& W. Schnotz (Eds.), Understanding multimedia documents (pp. 6178). New York: Springer.

Boltz, M. (1992). Temporal accent structure and the remembering of filmed narratives. Journal of Experimental Psychology: Human Perception and Performance, 18, 90-105.

Boucheix, J. M., \& Guignard, H. (2005). What animated illustrations conditions can improve technical document comprehension in young students? Format, signaling and control of the presentation. European Journal of Psychology of Education, 20, 369-388.

Catrambone, R. (1995). Aiding subgoal learning: Effects on transfer. Journal of Education \& Psychology, 87, 5-17.

Catrambone, R. (1996). Generalizing solution procedures learned from examples. Journal of Experimental Psychology. Learning, Memory, and Cognition, 22, 1020-1031.

Catrambone, R. (1998). The subgoal learning model: Creating better examples so that students can solve novel problems. Journal of Experimental Psychology: General, 127, 335-376.

Chandler, P. (2009). Dynamic visualizations and hypermedia: Beyond the "Wow" factor. Computers in Human Behavior, 25, 389-392.

De Koning, B. B., Tabbers, H. K., Rikers, R. M. J. P., \& Paas, F. (2009). Towards a framework for attention cueing in instructional animations: Guidelines for research and design. Educational Psychology Review, $21,113-140$.

Hanson, C., \& Hirst, W. (1989). On the representation of events: A study of orientation, recall, and recognition. Journal of Experimental Psychology: General, 118, 136-147. 
Hard, B. M., Tversky, B., \& Lang, D. (2006). Making sense of abstract events: Building event schemas. Memory \& Cognition, 34, 1221-1235.

Hasler, B. S., Kersten, B., \& Sweller, J. (2007). Learner control, cognitive load and instructional animation. Applied Cognitive Psychology, 21, 713-729.

Hegarty, M., Kriz, S., \& Cate, C. (2003). The roles of mental animation and external animation in understanding mechanical systems. Cognition and Instruction, 21, 325-360.

Höffler, T. N., \& Leutner, D. (2007). Instructional animation versus static pictures: A meta-analysis. Learning and Instruction, 17, 722-738.

Kahneman, D., \& Beatty, J. (1966). Pupil diameter and load on memory. Science, 154, 1583-1585.

Kalyuga, S. (2007). Expertise reversal effect and its implications for learner-tailored instruction. Educational Psychology Review, 19, 509-539.

Koopman, C., \& Newtson, D. (1981). Level of analysis in the perception of ongoing instruction: An exploratory study. Journal of Education \& Psychology, 73, 212-223.

Kurby, C. A., \& Zacks, J. M. (2008). Segmentation in the perception and memory of events. Trends in Cognitive Sciences, 12, 72-79.

Lowe, R. K. (1999). Extracting information from an animation during complex visual learning. European Journal of Psychology of Education, 14, 225-244.

Mayer, R. E., \& Chandler, P. (2001). When learning is just a click away: Does simple user interaction foster deeper understanding of multimedia messages? Journal of Education \& Psychology, 93, 390-397.

Mayer, R. E., Dow, G. T., \& Mayer, S. (2003). Multimedia learning in an interactive self-explaining environment: What works in the design of agent-based microworlds? Journal of Education \& Psychology, 95, 806-812.

Mayer, R. E., Hegarty, M., Mayer, S., \& Campbell, J. (2005). When static media promotes active learning: Annotated illustrations versus narrated animations in multimedia instruction. Journal of Experimental Psychology. Applied, 11, 256-265.

Mayer, R. E., \& Moreno, R. (2003). Nine ways to reduce cognitive load in multimedia learning. Educational Psychologist, 38, 43-52.

Meyer, K., Rasch, T., \& Schnotz, W. (2010). Effects of animation's speed of presentation on perceptual processing and learning. Learning and Instruction, 20, 136-145.

Moreno, R. (2007). Optimising learning from animations by minimising cognitive load: Cognitive and affective consequences of signalling and segmentation methods. Applied Cognitive Psychology, 21, 765781.

Moreno, R., \& Mayer, R. (2007). Interactive multimodal learning environments. Educational Psychology Review, 19, 309-326.

Moreno, R., \& Valdez, A. (2005). Cognitive load and learning effects of having students organize pictures and words in multimedia environments: The role of student interactivity and feedback. Educational Technology Research and Development, 53(3), 35-45.

Robroek, W. C. L., \& Van de Beek, G. (2005). Vaardigheden in de geneeskunde: Verbanden en verbinden [Skills in medicine: Bandages and bandaging techniques]. Maastricht, the Netherlands: Mediview.

Schnotz, W., \& Kürschner, C. (2007). A reconsideration of cognitive load theory. Educational Psychology Review, 19, 460-508.

Schnotz, W., \& Lowe, R. (2008). A unified view of learning from animated and static graphics. In R. Lowe \& W. Schnotz (Eds.), Learning with animations: Research implications for design (pp. 304-356). New York: Cambridge University Press.

Schnotz, W., \& Rasch, T. (2005). Enabling, facilitating, and inhibiting effects of animations in multimedia learning: Why reduction of cognitive load can have negative results on learning. Educational Technology Research and Development, 53, 47-58.

Schwan, S., \& Garsoffky, B. (2004). The cognitive representation of filmic event summaries. Applied Cognitive Psychology, 18, 37-55.

Schwan, S., Garsoffky, B., \& Hesse, F. W. (2000). Do film cuts facilitate the perceptual and cognitive organization of activity sequences? Memory \& Cognition, 28, 214-223.

Schwan, S., Hesse, F. W., \& Garsoffky, B. (1998). The relationship between formal filmic means and the segmentation behavior of film viewers. Journal of Broadcasting \& Electronic Media, 42, 237-249.

Sommerville, J. A., \& Decety, J. (2006). Weaving the fabric of social interaction: Articulating developmental psychology and cognitive neuroscience in the domain of motor cognition. Psychonomic Bulletin \& Review, 13, 179-200.

Spanjers, I. A. E., Wouters, P., Van Gog, T., \& Van Merriënboer, J. J. G. (2010). An expertise reversal effect of segmentation in learning from animations. Computers in Human Behavior (in press).

Swallow, K. M. \& Zacks, J. M. (2004). Hierarchical grouping of events revealed by eye movements. Poster presented at 45th annual Meeting of the Psychonomic Society,.Minneapolis, MN. 
Sweller, J. (2010). Element interactivity and intrinsic, extraneous and germane cognitive load. Educational Psychology Review (this issue).

Sweller, J., Van Merriënboer, J. J. G., \& Paas, F. (1998). Cognitive architecture and instructional design. Educational Psychology Review, 10, 251-296.

Tversky, B., Bauer-Morrison, J., \& Bétrancourt, M. (2002). Animation: Can it facilitate? International Journal of Human Computer Studies, 57, 247-262.

Van Gerven, P. W. M., Paas, F., Van Merriënboer, J. J. G., \& Schmidt, H. G. (2004). Memory load and the cognitive pupillary response in aging. Psychophysiology, 41, 167-174.

Van Gog, T., Paas, F., Marcus, N., Ayres, P., \& Sweller, J. (2009). The mirror-neuron system and observational learning: Implications for the effectiveness of dynamic visualisations. Educational Psychology Review, 21, 21-30.

Vygotsky, L. S. (1978). Mind in society. Cambridge, MA: Harvard University Press.

Van Merriënboer, J. J. G., \& Sweller, J. (2005). Cognitive load theory and complex learning: Recent developments and future directions. Educational Psychology Review, 17, 147-177.

Wouters, P., Paas, F., \& Van Merriënboer, J. J. G. (2008). How to optimize learning from animated models: A review of guidelines based on cognitive load. Review of Educational Research, 78, 645-675.

Wouters, P., Tabbers, H. K., \& Paas, F. (2007). Interactivity in video-based models. Educational Psychology Review, 19, 327-342.

Zacks, J. M. (2004). Using movement and intentions to understand simple events. Cognitive Science, 28, 979-1008.

Zacks, J. M., Speer, N. K., Swallow, K. M., Braver, T. S., \& Reynolds, J. R. (2007). Event perception: A mind-brain perspective. Psychological Bulletin, 133, 273-293.

Zacks, J. M., Speer, N. K., Vettel, J. M., \& Jacoby, L. L. (2006). Event understanding and memory in healthy aging and dementia of the Alzheimer type. Psychology and Aging, 21, 466-482.

Zacks, J., Tversky, B., \& Iyer, G. (2001). Perceiving, remembering, and communicating structure in events. Journal of Experimental Psychology: General, 130, 29-58.

Zalla, T., Pradat-Diehl, P., \& Sirigu, A. (2003). Perception of action boundaries in patients with frontal lobe damage. Neuropsychologia, 41, 1619-1627. 